Tietje/Nowrot (Hrsg.)

Internationales Wirtschaftsrecht

De Gruyter Studium 



\section{Internationales Wirtschaftsrecht}

3., neu bearbeitete Auflage

Herausgegeben von

Dr. Christian Tietje, Professor an der Martin-Luther-Universität Halle-Wittenberg und

Dr. Karsten Nowrot, Professor an der Universität Hamburg

Bearbeitet von

Marc Bungenberg, Horst-Peter Götting, Urs Peter Gruber, Yoan Hermstrüwer, Anne Lauber-Rönsberg, Matthias Lehmann, Jörn Lüdemann, Andreas Maurer, Karsten Nowrot, Paul Oberhammer, Karsten Otte, Markus Pohlmann, August Reinisch, Alexander Rust, Sabine Schlemmer-Schulte, Rolf Sethe, Christian Tietje, Florian Wagner-von Papp, Friedl Weiss 
Zitiervorschlag: Tietje/Nowrot/Sethe/Lehmann Internationales Wirtschaftsrecht, § 15 Rn. 9

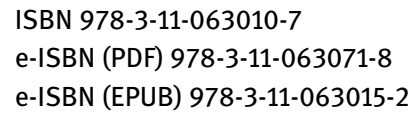

Library of Congress Control Number: 2021940484

\section{Bibliografische Information der Deutschen Nationalbibliothek}

Die Deutsche Nationalbibliothek verzeichnet diese Publikation in der Deutschen Nationalbibliografie; detaillierte bibliografische Daten sind im Internet über http://dnb.dnb.de abrufbar.

(c) 2022 Walter de Gruyter GmbH, Berlin/Boston Einbandabbildung: Gettyimages/DKosig Satz: jürgen ullrich typosatz, Nördlingen Druck und Bindung: $\mathrm{CPI}$ books $\mathrm{GmbH}$, Leck

www.degruyter.com 